\title{
Validation of PHITS Spallation Models from the Perspective of the Shielding Design of Transmutation Experimental Facility
}

\author{
Hiroki Iwamoto ${ }^{1, a}$ and Shin-ichiro Meigo ${ }^{1, b}$ \\ ${ }^{1}$ J-PARC Center, Japan Atomic Energy Agency, 2-4, Shirakata, Tokai-mura, Naka-gun, Ibaraki 319-1195, \\ Japan
}

\begin{abstract}
The impact of different spallation models implemented in the particle transport code PHITS on the shielding design of Transmutation Experimental Facility is investigated. For $400-\mathrm{MeV}$ proton incident on a lead-bismuth eutectic target, an effective dose rate at the end of a thick radiation shield (3-m-thick iron and 3-m-thick concrete) calculated by the Liège intranuclear cascade (INC) model version 4.6 (INCL4.6) coupled with the GEM code (INCL4.6/GEM) yields about twice as high as the Bertini INC model (Bertini/GEM). A comparison with experimental data for $500-\mathrm{MeV}$ proton incident on a thick lead target suggest that the prediction accuracy of INCL4.6/GEM would be better than that of Bertini/GEM. In contrast, it is found that the dose rates in beam ducts in front of targets calculated by the INCL4.6/GEM are lower than those by the Bertini/GEM. Since both models underestimate the experimental results for neutron-production doubledifferential cross sections at $180^{\circ}$ for $140-\mathrm{MeV}$ proton incident on carbon, iron, and gold targets, it is concluded that it is necessary to allow a margin for uncertainty caused by the spallation models, which is a factor of two, in estimating the dose rate induced by neutron streaming through a beam duct.
\end{abstract}

\section{Introduction}

To promote research, development, and demonstration of elemental technologies for acceleratordriven systems (ADS) [1-5], the Japan Atomic Energy Agency (JAEA) is planning to construct the Transmutation Experimental Facility (TEF) at J-PARC [6, 7]. In the construction of TEF, the Particle and Heavy Iron Transport code System, PHITS [8], plays an important role in the design of TEF shielding. Since the development of PHITS version 2.52, the Liège intranuclear cascade (INC) model version 4.6 (INCL4.6 [9]) has been employed as a default spallation model for the INC calculation in PHITS instead of the Bertini INC model [10]. Although, within the framework of the International Atomic Energy Agency (IAEA) benchmark program [11], a benchmark survey of spallation models demonstrated that the INCL model was the most accurate, further validation is still required to employ the INCL4.6 implemented in PHITS for specific applications such as ADS (see Ref. [5]).

This study investigates the impact of different spallation models, i.e., INCL4.6 and Bertini INC, on the TEF shielding characteristics. Here, the Generalized Evaporation Model (GEM) code [12] was

\footnotetext{
ae-mail: iwamoto.hiroki@jaea.go.jp

be-mail: meigo.shinichiro@jaea.go.jp
} 
employed for the de-excitation calculation following the intranuclear cascade calculation. Among various parameters on the shielding design such as effective dose rate, absorption dose rate, and radioactivity, we focus on the effective dose rate for representative apparatuses arranged in TEF; (1) a lead-bismuth eutectic (LBE) target and (2) a 10-W beam dump for beam commissioning. Another purpose of this study is to validate these models by comparing with experimental results, which were not included in the IAEA benchmark, from the perspective of the TEF shielding design.

\section{Outline of TEF and calculation conditions}

TEF utilizes a 250-kW proton beam accelerated with a 400-MeV LINAC accelerator at J-PARC. The proton beam is injected to the LBE target, in which intense spallation neutrons are produced. Basic concept of the TEF shielding design is the same as existing spallation neutron source facilities such as JSNS [13] at J-PARC, namely, the LBE target is surrounded by iron blocks, and an ordinary concrete shield is arranged outside the iron shield. To utilize the proton beam for various reactor-physics experiments with a subcritical assembly in TEF, a proton beam with powers up to $10 \mathrm{~W}$ is extracted from the $250-\mathrm{kW}$ proton beam, and the $10-\mathrm{W}$ beam dump for beam commissioning is installed in the TEF facility. Since a proton-beam duct is attached to the upstream side of each apparatus, estimation of the dose rate by neutron streaming through the beam duct is of importance.

Main characteristics of the LBE target and the 10-W beam dump are listed in Tables 1 and 2, respectively.

Table 1. Main characteristics of the LBE target.

\begin{tabular}{lcc}
\hline & unit & value \\
\hline Proton beam & & \\
Energy & $\mathrm{MeV}$ & 400 \\
Maximum power & $\mathrm{kW}$ & 250 \\
LBE target & & \\
Dimension & $\mathrm{cm}$ & $\mathrm{D} 15 \times \mathrm{L} 60$ \\
Density & $\mathrm{g} / \mathrm{cm}^{3}$ & 10.21 \\
\hline
\end{tabular}

Table 2. Main characteristics the $10-\mathrm{W}$ beam dump.

\begin{tabular}{lcc}
\hline & unit & value \\
\hline Proton beam & $\mathrm{MeV}$ & 400 \\
Energy & $\mathrm{W}$ & 10 \\
$\begin{array}{l}\text { Maximum power } \\
\text { Beam dump } \\
\text { Material }\end{array}$ & - & $\begin{array}{c}\text { graphite, iron, } \\
\text { and ordinary concrete }\end{array}$ \\
Dimension & $\mathrm{cm}$ & $20 \times 20 \times 40$ (graphite) \\
$\begin{array}{c}\text { Beam duct } \\
\text { Material }\end{array}$ & - & titanium \\
Inner diameter & $\mathrm{cm}$ & 10 \\
\hline
\end{tabular}

Figures 1 and 2 illustrate the PHITS calculation models of the LBE target and 10-W beam dump, respectively. The calculation was performed by PHITS version 2.76, and the nuclear data library JENDL-4.0 [14] was employed for the calculation of neutron-induced reactions below $20 \mathrm{MeV}$. The effective dose rates were evaluated by the use of dose conversion coefficients [15] implemented in PHITS. 


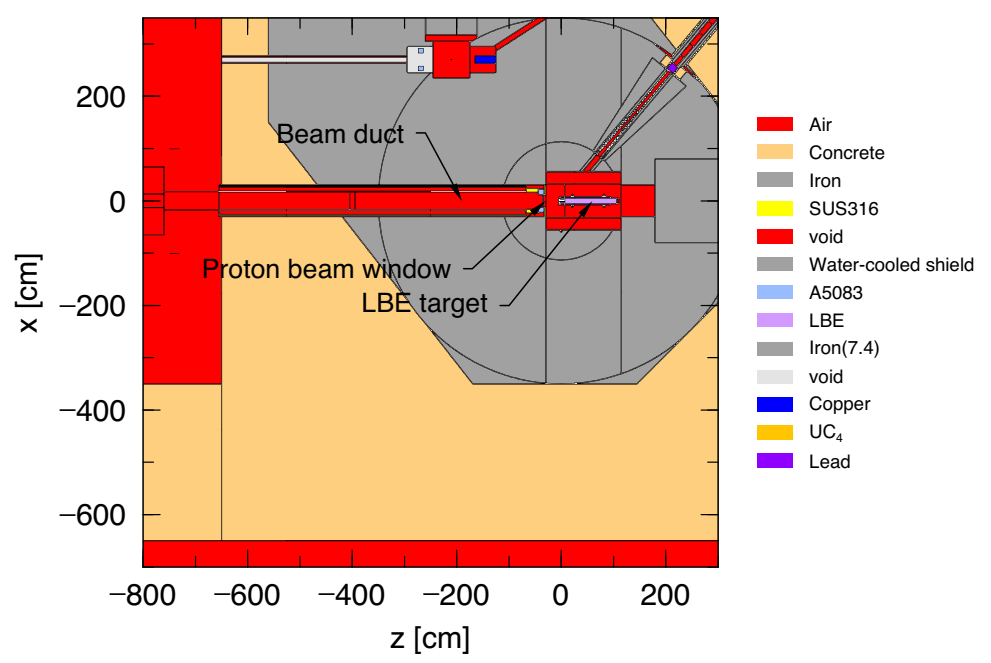

Figure 1. Calculation model of the LBE target.

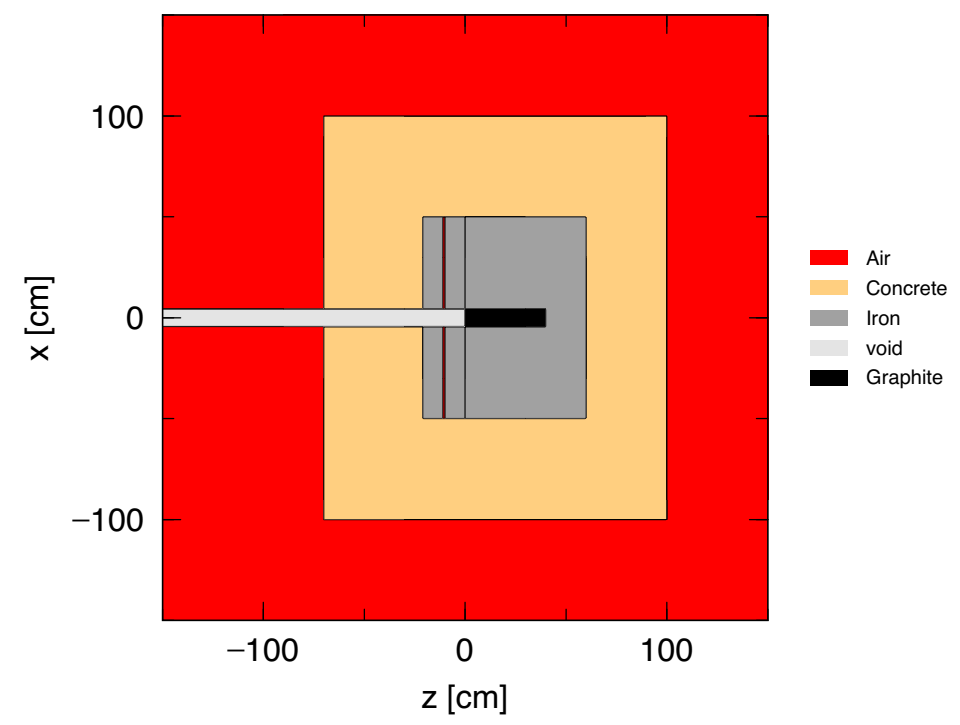

Figure 2. Calculation model of the $10-\mathrm{W}$ beam dump.

\section{Results and discussion}

\subsection{LBE target}

Figure 3 shows the effective dose rate in 3-m-thick iron and 3-m-thick concrete shields at $90^{\circ}$ from the beam direction. Here, the neutron contribution is divided into three components with resepct to its energy; thermal (below $0.4 \mathrm{eV}$ ), intermediate (0.4-1 MeV), and high-energy (above $1 \mathrm{MeV}$ ) neutrons. 


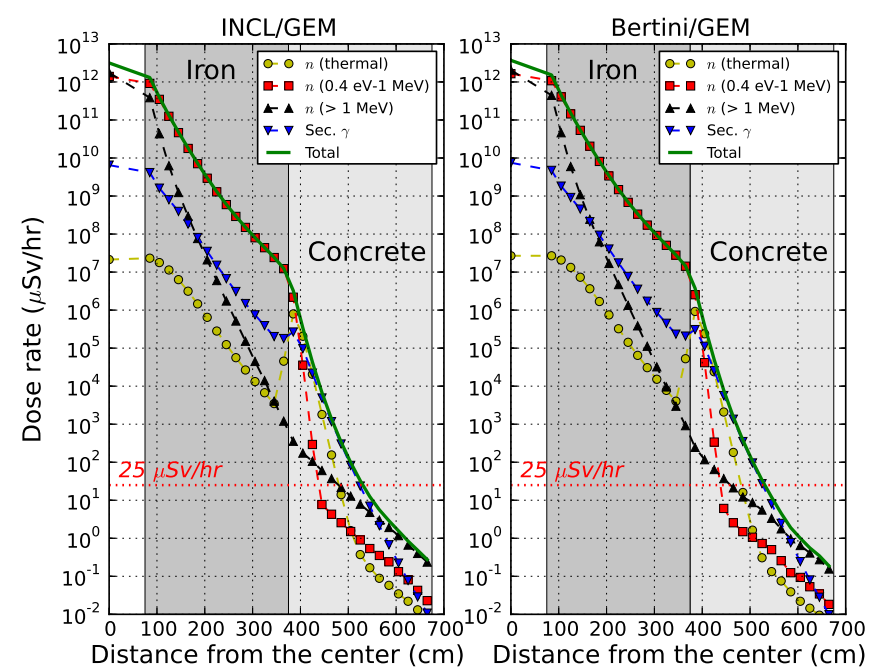

Figure 3. Effective dose rate in iron and concrete shields at $90^{\circ}$ from the beam direction.

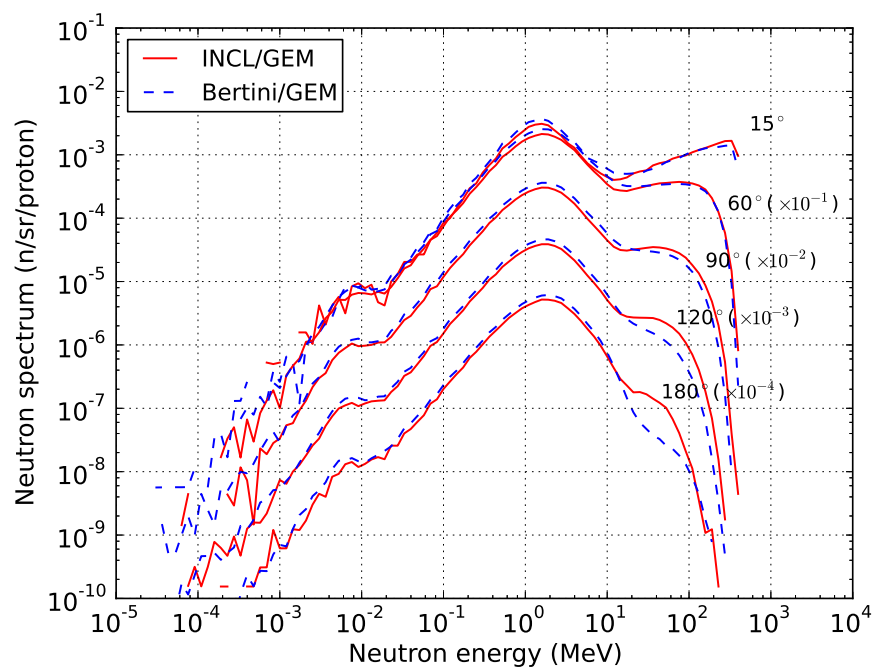

Figure 4. Neutron spectra produced from $400-\mathrm{MeV}$ proton incident on the LBE target.

The dose rate calculated by the INCL4.6/GEM is higher than that calculated by the Bertini/GEM. At the end of the iron and concrete shield, the INCL4.6/GEM calculation yields about twice as high as the Bertini/GEM, and the total values are dominated by the contribution of neutrons with energies above $1 \mathrm{MeV}$. Since both models uses the same total cross sections in simulating the particle transport, it appears that this difference mainly comes from the difference in neutrons produced from the LBE target. 


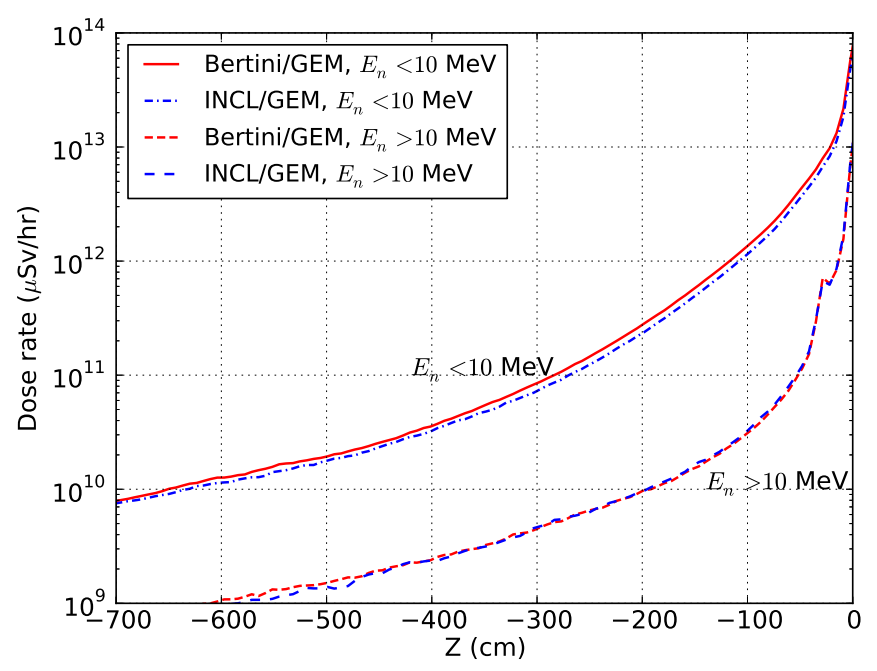

Figure 5. Effective dose rate at $180^{\circ}$ from the beam direction. The values of a coordinate on the horizontal axis are the same as those in Figure 1.

Figure 4 shows calculated neutron spectra produced from $400-\mathrm{MeV}$ proton incident on the LBE target for angles of $15^{\circ}, 60^{\circ}, 90^{\circ}, 120^{\circ}$, and $180^{\circ}$. The INCL4.6/GEM gives higher neutron spectra than the Bertini/GEM for energies above $10 \mathrm{MeV}$. This is the reason for the higher dose estimation by INCL4.6/GEM in Fig. 3.

Figure 5 shows the effective dose rate at $180^{\circ}$ from the beam direction. In contrast to the above results, the dose rate at $7 \mathrm{~m}$ away from the target calculated by the INCL4.6/GEM is slightly lower than that by the Bertini/GEM. This difference is attributable to the difference in low-energy neutrons produced from the LBE target (see Fig. 4).

\subsection{Beam Dump}

Figure 6 shows the effective dose rate around the beam dump for the beam direction. Figure 7 shows the double-differential cross sections at $180^{\circ}$ for $400-\mathrm{MeV}$ proton incident on a carbon target. As is the case with the LBE target, the INCL4.6/GEM gives lower values than the Bertini/GEM at the backward direction, which is due to the difference in neutron spectra for energies below $10 \mathrm{MeV}$ as shown in Fig. 7.

\subsection{Validation}

Figure 8 compares the thick target neutron yield for $500-\mathrm{MeV}$ protons on a $20-\mathrm{cm}$-thick lead target. Here, the experimental data are taken from Ref. [16]. INCL4.6/GEM is better suited for the spectral shape, whose prediction is within $1 \sigma$ experimental uncertainty over a wide energy range, whereas the Bertini/GEM underestimates the experiments for angles from $90^{\circ}$ to $150^{\circ}$.

Figure 9 compares the double-differential cross sections at $180^{\circ}$ for $140-\mathrm{MeV}$ protons on carbon, iron, and gold targets. The experimental data are taken from Ref. [17]. Although the incident energy is 


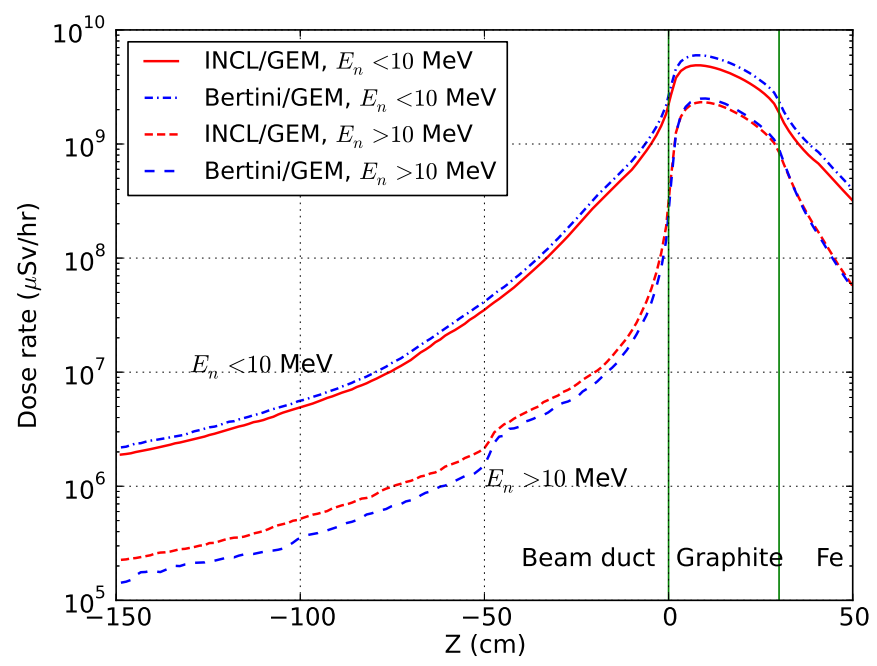

Figure 6. Effective dose rate around the beam dump for the beam directions. The values of a coordinate on the horizontal axis are the same as those in Figure 2.

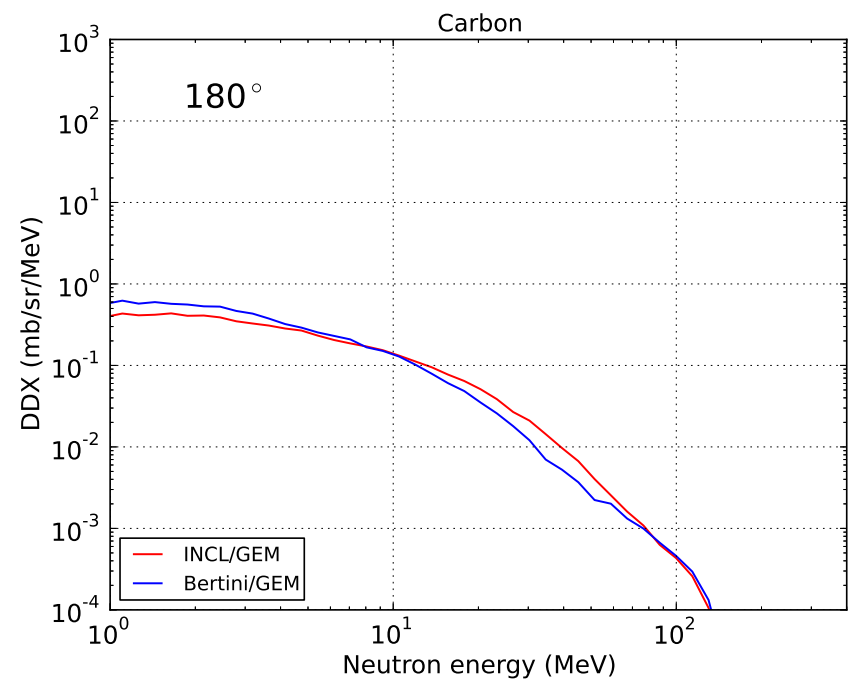

Figure 7. Double-differential cross sections at $180^{\circ}$ for $400-\mathrm{MeV}$ proton incident on a carbon target.

different, the trends of spectral shape are the same as those for the $400-\mathrm{MeV}$ proton incident reaction (see Fig. 7). It was found from Fig. 9 that both models underestimate the experiments overall and fail to reproduce the spectral shape. This underestimation, especially at energies below $10 \mathrm{MeV}$, would cause an underestimation of the effective dose rate for backscattered neutrons. Since neutrons with energies below $10 \mathrm{MeV}$ at the backward directions are mainly produced by the de-excitation 

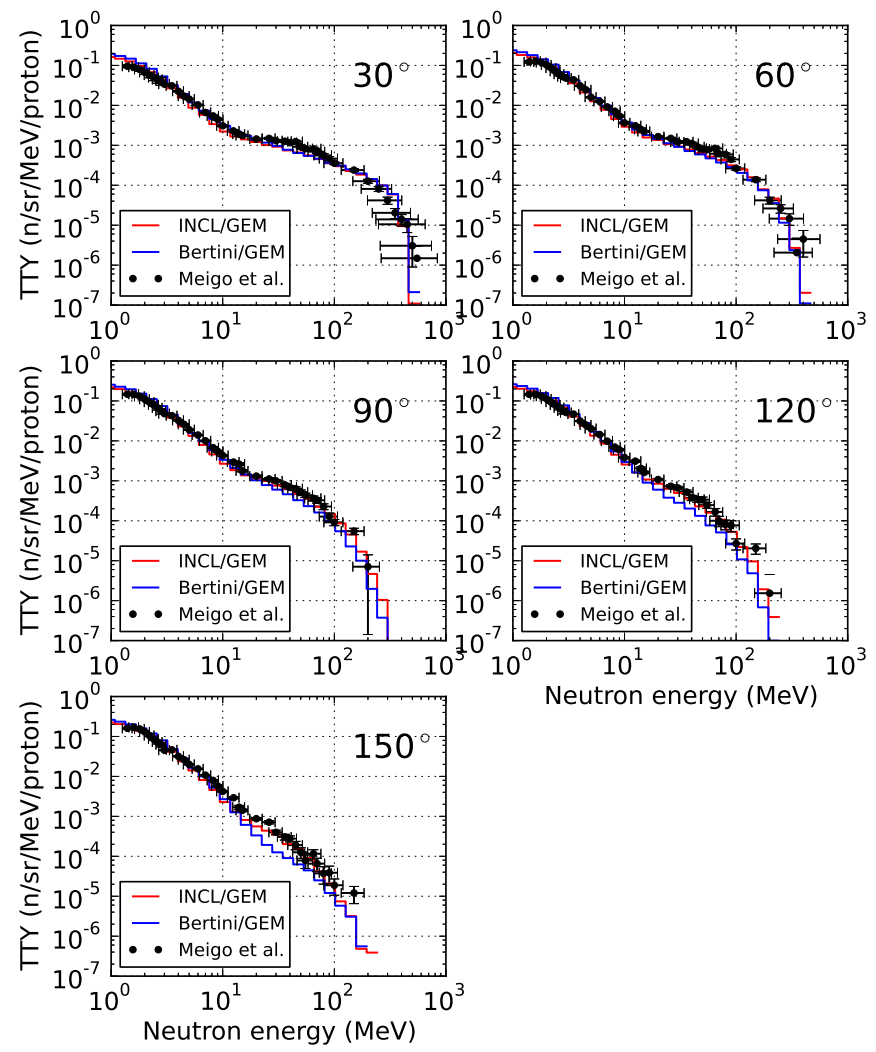

Figure 8. Comparison of thick target neutron yield for $500-\mathrm{MeV}$ protons on 20 -cm-thick lead.

process, the results indicate that modification or improvement of the de-excitation model, GEM, will be necessary.

\section{Conclusion}

In this study, the impact of different spallation models (i.e., INCL4.6/GEM and Bertini/GEM) on the TEF shielding design was investigated. The results showed that the INCL4.6/GEM estimates the dose rate at the end of the radiation shield $\sim 1.5$ times higher than the Bertini/GEM, whereas the INCL4.6/GEM calculation gives lower dose rate than the Bertini/GEM at $180^{\circ}$ for the LBE target and the 10-W beam dump. The comparison with the experiment of Ref. [16] showed that the INCL4.6/GEM calculation is more reliable for the TEF shielding design. In contrast, for energies below $10 \mathrm{MeV}$, both spallation models underestimate the neutron spectra at $180^{\circ}$ in Ref. [17]. This indicates that the PHITS spallation models could underestimate the dose rate for the backward directions. While it is difficult to derive a conclusion only from the limited experimental results, it appears 

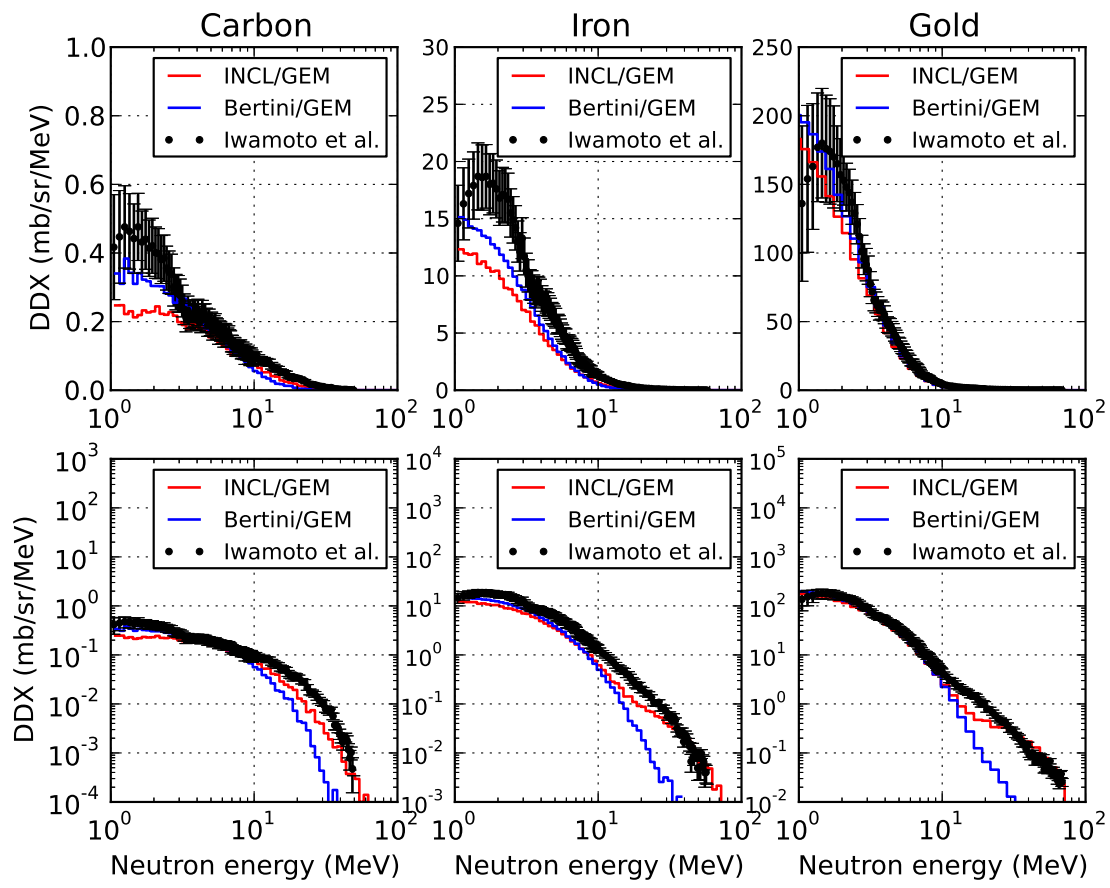

Figure 9. Comparison of double-differential cross sections at $180^{\circ}$ for $140-\mathrm{MeV}$ proton incident on carbon, iron, and gold targets. Upper and lower panels indicate linear and logarithmic scales on the vertical axis, respectively.

that uncertainty in the effective dose rate at the $180^{\circ}$ direction induced by spallation models would be within a factor of two. Therefore, in calculating the dose rate by backscattered neutrons, a margin of this uncertainty should be considered. Thus, the PHITS spallation models still have room for improvement especially for neutron emissions at the backward angles. To achieve more reliable dose estimation, systematic experiments on the backward neutron data is needed.

\section{References}

[1] K. Tsujimoto et al., Nuclear Technology 161, 315-328 (2008)

[2] K. Nishihara et al., Journal of Nuclear Science and Technology 45, 812-822 (2008)

[3] T. Sugawara et al., Journal of Nuclear Science and Technology 47, 953-962 (2008)

[4] H. Iwamoto et al., Journal of Nuclear Science and Technology 50, 856-862 (2013)

[5] H. Iwamoto et al., Journal of Nuclear Science and Technology 53, 1585-1594 (2016)

[6] K. Tsujimoto et al., Journal of Nuclear Science and Technology 44, 483-490 (2007)

[7] T. Sasa and H. Oigawa, Plasma and Fusion Research 9, 4401113 (2014)

[8] T. Sato et al., Journal of Nuclear Science and Technology 50, 913-923 (2013)

[9] A. Boudard et al., Physical Review C 87, 014606 (2013)

[10] H. W. Bertini, Physical Review 131, 1801-1821 (1963)

[11] J. C. David et al., Progress in Nuclear Science and Technology 2, 942-947 (2011) 
[12] S. Furihata, Nuclear Instruments and Methods in Physics Research, Section B 171, 251-258 (2000)

[13] F. Maekawa et al., Nuclear Instruments and Materials in Physics Research, Section A 620, 259265 (2010)

[14] K. Shibata et al., Journal of Nuclear Science and Technology 48, 1-30 (2011)

[15] Y. Sakamoto et al., Dose conversion coefficients for high-energy photons, electrons and protons (Japan Atomic Energy Research Institute, Tokai, 2003) JAERI-1345

[16] S. Meigo et al., Nuclear Instruments and Methods in Physics Research, Section A 431, 521-530 (1999)

[17] Y. Iwamoto et al., Journal of the Korean Physical Society 59, 1753-1756 (2011) 